\title{
EXPLORING LIMIT BEHAVIOUR OF NON-QUADRATIC TERMS VIA H-MEASURES. APPLICATION TO SMALL AMPLITUDE HOMOGENISATION.
}

\author{
MARTIN LAZAR
}

\begin{abstract}
A method is developed for analysing asymptotic behaviour of terms involving an arbitrary integer order powers of $\mathrm{L}^{p}$ functions by means of H-measures. It is applied to the small amplitude homogenisation problem for a stationary diffusion equation, in which coefficients are assumed to be analytic perturbations of a constant, enabling formulæ for higher order correction terms in a general, non-periodic setting. Explicit expressions in terms of Fourier coefficients are obtained under periodicity assumption. The method enables its generalisation and application to the corresponding non-stationary equation, as well as to some other small amplitude homogenisation problems.
\end{abstract}

\section{INTRODUCTION AND MOTIVATION}

H-measures, as originally introduced a quarter of century ago by L. Tartar [17] and (independently) P. Gérard [10] are kind of a microlocal defect tool, measuring deflection of weak from strong $\mathrm{L}^{2}$ convergence. They explore quadratic limit behaviour of bounded $\mathrm{L}^{2}$ sequences.

A prominent feature of $\mathrm{H}$-measures is their capability to keep track of an equation satisfied by functions generating them. More precisely, if an $\mathrm{H}$-measure is associated to solutions of an equation $P u_{n}=0$ (accompanied by a series of initial/boundary conditions), one can take advantage of their basic properties: the localisation and the propagation one. The former constrains the support of the H-measure within the characteristic set of the (pseudo) differential operator $P$, while the latter states that the measure (as well as concentration and oscillation effects) propagates along bicharacteristics of $P$.

Since their introduction, they have been successfully applied in many mathematical fields - let us here mention generalisation of compensated compactness results to equations with variable coefficients [10,17], applications in the control theory $[9,16]$, the velocity averaging results $[10,13]$, as well as explicit formulæ and bounds in homogenisation $[17,4,5]$.

Original $\mathrm{H}$-measures are restricted to the $\mathrm{L}^{2}$ framework. This constraint has partially been overcome with the introduction of H-distributions [7] - a generalisation of the concept to the $\mathrm{L}^{p}, p \geq 1$ framework. More precisely, a new tool is constructed with the aim of exploring products of a form $\int u_{n} v_{n}$, with a sequence $\left(u_{n}\right)$ being bounded in $\mathrm{L}^{p}$, while $v_{n}$ are taken from the corresponding dual $\mathrm{L}^{p^{\prime}}$

Date: January 27, 2016.

2010 Mathematics Subject Classification. 35B27, 35J15, 35S05.

Key words and phrases. small amplitude homogenisation, H-measures, non-quadratic terms. 
However, the above generalisation is still bound to a study of quadratic terms (with possibly different factors), and does not handle higher order terms, such as cubic ones. The aim of this paper is to investigate possibilities of handling a general $\mathrm{L}^{p}, p \geq 2$ sequences and to describe, roughly speaking, a limit behaviour of $\int\left|u_{n}\right|^{p}$. Here it should be mentioned that actual products we deal with are more complicated, allowing each factor to be accompanied by a pseudodifferential operator acting on it.

The analysis below is based on a relatively simple idea: for a sequence of functions $u_{n}$ converging weakly to zero in $\mathrm{L}^{p}, p \geq 2$, the sequence $\left(u_{n}^{p / 2}\right)$ is bounded in $\mathrm{L}^{2}$, and determines (up to a subsequence) an H-measure denoted by $\mu_{p / 2}$. Thus the limit

of $\int\left|u_{n}\right|^{p}=\int u_{n}^{p / 2} \overline{u_{n}^{p / 2}}$ can be expressed by the measure $\mu_{p / 2}$, which in some cases can be calculated explicitly. Of course, one has to be aware that in the absence of strong convergence, the weak limit of $u_{n}^{p / 2}$ in general differs from zero, which requires correction terms entering the expression.

The obtained results are applied to a small amplitude homogenisation problem. The main idea of a small amplitude approximation consists of taking a (formal) expansion of a solution to a problem under consideration with respect to a small parameter representing perturbation of the coefficient. Originally introduced by L. Tartar in [17] for a stationary diffusion problem, the approach has subsequently been elaborated and applied to more general homogenisation [4, 8], optimal design $[1,2]$ and inverse problems [12]. In all these papers $\mathrm{H}$-measures are used as the main analytical tool, and the analysis is performed up to the second order expansion, within which the result is obtained by exploring limits of quadratic terms. Handling of non-quadratic terms, appearing in higher order expansion, is, however, not achievable by a standard usage of $\mathrm{H}$-measures and requires a different approach. In this paper we try to make a step forward in that direction and to obtain expressions for higher order correction terms.

The paper is organised as follows. In the next section we describe in details the above presented idea, and demonstrate the method of expressing limit of higher order terms via (original) H-measures associated to an appropriate combination of given $\mathrm{L}^{p}$ functions. Application of the method is demonstrated in Section 3 on the example of small amplitude homogenisation for a stationary diffusion problem, with a particular intention given to a special, but important case of periodic functions. The paper is closed by some concluding remarks, and by pointing toward some related and open problems.

\section{EXPLORING NON-QUADRATIC TERMS THROUGH H-MEASURES}

2.1. General setting. The original H-measures explore quadratic limit behaviour of $\mathrm{L}^{2}$ terms, and their existence theorem can be formulated as follows (cf. [10, 17]).

Theorem 1. Let $\left(u_{n}\right)$ be a sequence converging weakly to zero in $\mathrm{L}^{2}\left(\mathbf{R}^{d}\right)$. Then, after passing to a subsequence (not relabeled), there exists a nonnegative Radon measure $\mu$ on the cospherical bundle $\mathbf{R}^{d} \times \mathrm{S}^{d-1}$ such that for every $\varphi_{1}, \varphi_{2} \in \mathrm{C}_{0}\left(\mathbf{R}^{d}\right)$ 
and $\psi \in \mathrm{C}\left(\mathrm{S}^{d-1}\right)$, it holds

$$
\begin{aligned}
\lim _{n \rightarrow \infty} \int_{\mathbf{R}^{d}} \mathcal{A}_{\psi}\left(\varphi_{1} u_{n}\right)(\mathbf{x}) \overline{\left(\varphi_{2} u_{n}\right)(\mathbf{x})} d \mathbf{x} & =\left\langle\mu,\left(\varphi_{1} \overline{\varphi_{2}}\right) \otimes \psi\right\rangle \\
& =\int_{\mathbf{R}^{d} \times S^{d-1}} \varphi_{1}(\mathbf{x}) \overline{\varphi_{2}}(\mathbf{x}) \psi(\boldsymbol{\xi}) d \mu(\mathbf{x}, \boldsymbol{\xi}) .
\end{aligned}
$$

where $\mathcal{A}_{\psi}$ is the (Fourier) multiplier operator on $\mathbf{R}^{d}$ associated to $\psi(\boldsymbol{\xi} /|\boldsymbol{\xi}|)$.

The measure $\mu$ is called the H-measure associated to the (sub)sequence $\left(\mathbf{u}_{n^{\prime}}\right)$. In the sequel we shall often abused terminology and notation by assuming that we have already passed to a subsequence determining an H-measure.

Notation. Throughout the paper by $\langle\cdot, \cdot\rangle$ stands for a sesquilinear dual product, taken to be antilinear in the first, while linear in the second variable. By $\nabla$ we denote the tensor product of functions in different variables.

The theorem also generalises to the vector sequences $\left(\mathbf{u}_{n}\right)$, resulting in a hermitian, positive semi-definite matrix H-measure, whose diagonal elements are (scalar) H-measures associated to a corresponding component $u_{n}^{i}$.

The proof of the last theorem is based on the (First) commutation lemma [17, Lemma 1.7], enabling exchange of the multiplier operator $A_{\psi}$ and the operator of multiplication by $\varphi_{i}$ in (1) when passing to the limit. Thus the limit depends on the product $\varphi_{1} \varphi_{2}$ only and results in a bilinear functional on $\mathrm{C}_{0}\left(\mathbf{R}^{d}\right) \otimes \mathrm{C}\left(\mathrm{S}^{d-1}\right)$. Taking into account its positivity, and by using the Schwartz kernel theorem, as well as the Schwartz lemma on nonnegative distributions, one shows that the functional is a Radon measure in both variables, $\mathbf{x}$ and $\boldsymbol{\xi}$.

A multiplier operator $A_{\psi}$ associated to a bounded symbol $\psi$ is a continuous operator on $\mathrm{L}^{2}\left(\mathbf{R}^{d}\right)$, which is easily demonstrated by means of the Fourier transform. Generalisation of that result to an $\mathrm{L}^{p}$ setting is provided by the Marcinkiewicz multiplier theorem (e.g. [11, Theorem 5.2.4] showing that $A_{\psi}$ is a bounded operator on $\mathrm{L}^{p}\left(\mathbf{R}^{d}\right)$, for any $p \in\langle 1, \infty\rangle$ and $\psi$ of class $\mathrm{C}^{d}$.

We would like to generalise Theorem 1 by considering higher order expressions in $u_{n} \in \mathrm{L}^{p}, p \in \mathbf{N}$. More precisely we are interested in expressing the limit of

$$
\int_{\mathbf{R}^{d}} \mathcal{A}_{\psi_{1}}\left(\varphi_{1} u_{n}\right)(\mathbf{x}) \mathcal{A}_{\psi_{2}}\left(\varphi_{2} u_{n}\right)(\mathbf{x}) \ldots \mathcal{A}_{\psi_{p}}\left(\varphi_{p} u_{n}\right)(\mathbf{x}) d \mathbf{x}
$$

where $\varphi_{i}, \psi_{i}, i=1$..p are appropriate test functions. The following result holds.

Theorem 2. Let $\left(u_{n}\right)$ be a sequence converging weakly to zero in $\mathrm{L}_{\text {loc }}^{p+\varepsilon}\left(\mathbf{R}^{d}\right)$ for some $p \in \mathbf{N}$ and $\varepsilon>0$. Then for any choice of test functions $\varphi_{i} \in \mathbf{C}_{c}\left(\mathbf{R}^{d}\right), \psi_{i} \in$ $\mathrm{C}^{d}\left(\mathrm{~S}^{d-1}\right), i=1 . . p$ it holds

$$
\lim _{n} \int_{\mathbf{R}^{d}} \mathcal{A}_{\psi_{1}}\left(\varphi_{1} u_{n}\right)(\mathbf{x}) \cdot \ldots \cdot \mathcal{A}_{\psi_{p}}\left(\varphi_{p} u_{n}\right)(\mathbf{x}) d \mathbf{x}=\left\langle\mu_{v w}, \varphi \otimes 1\right\rangle+\int(\varphi v)(\mathbf{x}) \bar{w}(\mathbf{x}) d \mathbf{x}
$$

where $\varphi=\prod_{i=1}^{p} \varphi_{i}$, while $\mu_{v w}$ is the off-diagonal component of the matrix $H$ measure associated to the sequence $\left(v_{n}-v, w_{n}-w\right)$ defined by (5) and (6) below.

Proof: By the theorem assumptions, the products $\varphi_{i} u_{n}$ are bounded in $\mathrm{L}^{p+\varepsilon}\left(\mathbf{R}^{d}\right)$ for all $i=1$..p. Additionally the Marcinkiewicz multiplier theorem provides boundedness of $\mathcal{A}_{\psi_{i}}\left(\varphi_{i} u_{n}\right)$ in the same space, as well. As by the (commutation ) Lemma 3 
the commutator $C_{i}=\mathcal{A}_{\psi_{i}} \varphi_{i}-\varphi_{i} \mathcal{A}_{\psi_{i}}$ is a compact operator on $\mathrm{L}^{p}\left(\mathbf{R}^{d}\right)$, we can consecutively exchange the order of operators in (3), showing that the corresponding limit equals

$$
\lim _{n} \int_{\mathbf{R}^{d}} \varphi \mathcal{A}_{\psi_{1}}\left(\chi_{1} u_{n}\right)(\mathbf{x}) \cdot \ldots \cdot \mathcal{A}_{\psi_{p}}\left(\chi_{p} u_{n}\right)(\mathbf{x}) d \mathbf{x}=\lim _{n} \int_{\mathbf{R}^{d}} \varphi v_{n} \overline{w_{n}} d \mathbf{x} .
$$

where the last two factors are defined by

$$
v_{n}:=\left\{\begin{array}{c}
\prod_{i=1}^{p / 2} \mathcal{A}_{\psi_{i}}\left(\chi_{i} u_{n}\right), \quad p \in 2 \mathbf{N} \\
\prod_{i=1}^{(p-1) / 2} \mathcal{A}_{\psi_{i}}\left(\chi_{i} u_{n}\right)\left(\mathcal{A}_{\psi_{(p+1) / 2}}\left(\chi_{(p+1) / 2} u_{n}\right)\right)^{1 / 2}, \quad \text { elsewhere }
\end{array}\right.
$$

and

$$
\overline{w_{n}}:=\left\{\begin{array}{c}
\prod_{i=p / 2+1}^{p} \mathcal{A}_{\psi_{i}}\left(\chi_{i} u_{n}\right), \quad p \in 2 \mathbf{N} \\
\left(\mathcal{A}_{\psi_{(p+1) / 2}}\left(\chi_{(p+1) / 2} u_{n}\right)\right)^{1 / 2} \prod_{i=(p+3) / 2}^{p} \mathcal{A}_{\psi_{i}}\left(\chi_{i} u_{n}\right), \quad \text { elsewhere. }
\end{array}\right.
$$

In the above $\chi_{i}$ stands for the characteristic function of the support of $\varphi_{i}$. The conjugation sign originate from the scalar product used in the definition of $\mathrm{H}$ measures (1). We also assume that one branch of the square root has been selected (the choice does not effect the result).

Sequences $\left(v_{n}\right)$ and $\left(w_{n}\right)$ are bounded in $\mathrm{L}^{2}\left(\mathbf{R}^{d}\right)$, and (after possible passing to a subsequence) we denote their weak $\mathrm{L}^{2}$ limits by $v$ and $w$, respectively. Note that in the absence of the strong (zero) convergence of $\left(u_{n}\right)$, the limits $v$ and $w$ in general differ from zero.

Rewriting the last term in (4) as

$$
\lim _{n} \int_{\mathbf{R}^{d}} \varphi\left(v_{n}-v\right) \overline{\left(w_{n}-w\right)} d \mathbf{x}+\int_{\mathbf{R}^{d}} \varphi v \bar{w} d \mathbf{x},
$$

and expressing the last limit via H-measure $\mu_{v w}$ one obtains the result.

The generalisation of the First commutation lemma to the non- $\mathrm{L}^{2}$ framework can be found in [7], whose (slightly corrected) version we reproduce here.

Lemma 3. Let $\left(u_{n}\right)$ be a bounded sequence of functions in $\mathrm{L}^{2}\left(\mathbf{R}^{d}\right) \cap \mathrm{L}^{p}\left(\mathbf{R}^{d}\right)$ for some $p \in\langle 2, \infty]$, converging weakly to zero (in the sense of distributions). Denote by $C=\mathcal{A}_{\psi} \varphi-\varphi \mathcal{A}_{\psi}$ the commutator determined by $\varphi \in \mathrm{C}_{0}\left(\mathbf{R}^{d}\right)$ and $\psi \in \mathrm{C}^{d}\left(\mathrm{~S}^{d-1}\right)$. Then the sequence $\left(C u_{n}\right)$ converges to zero strongly in $\mathrm{L}^{q}\left(\mathbf{R}^{d}\right)$ for any $q \in[2, p\rangle$.

The proof of the lemma is based on the interpolation inequality, the First commutation lemma ([17, Lemma 1.7], providing compactness of the commutator $C$ on $\mathrm{L}^{2}\left(\mathbf{R}^{d}\right)$ ), and the Marcinkiewicz multiplier theorem (providing boundedness of the multiplier $\mathcal{A}_{\psi}$ on $\mathrm{L}^{q}\left(\mathbf{R}^{d}\right)$ for any $q \in\langle 1, \infty\rangle$ and $\psi$ smooth enough).

Remark 4. - The additional regularity assumptions on $\left(u_{n}\right)$ (requiring it to belong to $\mathrm{L}^{p+\varepsilon}\left(\mathbf{R}^{d}\right)$ for some positive $\varepsilon$ ) and $\psi$ (assuming it to be of class $\mathrm{C}^{d}$ ) are necessary for the application of the (commutation) Lemma 3. It enables the limit in (3) to depend on the product $\varphi$ of test functions $\varphi_{i}$ only. 
- Note that by relation (4) it follows that the limit in (3) defines a continuous $(p+1)$-linear form $B$ on $\mathrm{C}_{0}\left(\mathbf{R}^{d}\right) \times \mathrm{C}^{d}\left(\mathrm{~S}^{d-1}\right) \times \ldots \times \mathrm{C}^{d}\left(\mathrm{~S}^{d-1}\right)$. In the special case of $p=2$ one can use Plancherel theorem in order to get a bilinear form depending on products $\varphi=\prod_{i} \varphi_{i}$ and $\psi=\prod_{i} \psi_{i}$ only, resulting in a distribution, and eventually an (H-)measure on $\mathbf{R}^{d} \times \mathrm{S}^{d-1}$. Usually this tool is not at our disposal, and we are left with a more general object. However, relation (3) shows that the object is a measure with respect to $\mathbf{x}$ variable. More precisely, for any choice of test functions $\psi_{i}, i=1 . . p$, the functional $B\left(\cdot, \psi_{1}, \ldots, \psi_{p}\right)$ equals the measure $\mu_{v w}+v \bar{w} \lambda(\mathbf{x})$, with the last term standing for the Lebesgue measure.

- The last theorem can be adapted in order to compute limits of more generalised expressions of the form (for simplicity of the notation we restrict to the even $p$ ):

$$
\begin{aligned}
\int_{\mathbf{R}^{d}} \mathcal{A}_{\psi}\left(\mathcal{A}_{\psi_{1}}\left(\varphi_{1} u_{n}\right) \cdot \ldots \cdot \mathcal{A}_{\psi_{p / 2}}\left(\varphi_{p / 2} u_{n}\right)\right)(\mathbf{x}) \\
\cdot \mathcal{A}_{\psi_{p / 2+1}}\left(\varphi_{p / 2+1} u_{n}\right) \cdot \ldots \cdot \mathcal{A}_{\psi_{p}}\left(\varphi_{p} u_{n}\right)(\mathbf{x}) d \mathbf{x} .
\end{aligned}
$$

The difference compared to (3) is in an additional multiplier (with symbol $\psi$ ) acting on half of the factors.

Carefully repeating steps of the proof of Theorem 2 one obtains that the limit of (7) equals

$$
\left\langle\mu_{v w}, \varphi \otimes \psi\right\rangle+\int \mathcal{A}_{\psi}\left(\varphi_{1} \cdot \ldots \cdot \varphi_{p / 2} v\right)(\mathbf{x}) \overline{\varphi_{p / 2+1} \cdot \ldots \cdot \varphi_{p} w}(\mathbf{x}) d \mathbf{x}
$$

the result coinciding with (3) for $\psi=1$.

2.2. Localisation principle. As already stated in the introduction, one of the most important properties of $\mathrm{H}$-measures is the so called localisation property (e.g. [10, Corollary 2.2]), playing a crucial role in most of successful applications of the tool. Thus it would be important to check if the same principle applies when analysing limit behaviour of higher order terms. Here we substantiate a positive answer for an $\mathrm{L}^{\infty}$ sequence, but it holds for more general situations as well.

Suppose $u_{n} \in \mathrm{L}^{\infty}\left(\mathbf{R}^{d}\right)$ are solutions to an equation $P u_{n}=0$, converging vaguely to zero. By the localisation principle, the associated H-measure is supported within the characteristic set of the operator $P$. But the same constraint also applies for off-diagonal terms of a matrix H-measure associated to a vector sequence $\left(u_{n}, w_{n}\right)$, with $\left(w_{n}\right)$ being an arbitrary bounded $\mathrm{L}^{2}$ sequence.

For that reason, we can split the integrand in (2) into two parts, the first one coinciding with the first factor, while the second one containing the rest of them, i.e. $w_{n}=\prod_{i=2}^{p} \mathcal{A}_{\psi_{i}}\left(\varphi_{i} u_{n}\right)$. Taking into account Remark 4 (the last item), we get the limit of (2) equals $\left\langle\mu_{u w}, \varphi \otimes \psi_{1}\right\rangle$, where $\mu_{u w}$ is an off-diagonal term of an $\mathrm{H}$ measure associated to $\left(u_{n}, w_{n}-w\right)$. In accordance to above, it satisfies the same constraints as an H-measure associated to $\left(u_{n}\right)$, i.e. its support is localised within the characteristic set of $P$.

2.3. Explicit formulæ in the periodic setting. Let $\left(u_{n}\right)$ be a bounded sequence of periodic functions in $\mathrm{L}_{\mathrm{loc}}^{\infty}\left(\mathbf{R}^{d}\right)$ defined by

$$
u_{n}(\mathbf{x})=\sum_{\mathbf{k} \in \mathbf{Z}^{d}} \hat{u}_{\mathbf{k}} e^{2 \pi i n \mathbf{k} \cdot \mathbf{x}} .
$$


Hereby we assume the mean value $\hat{u}_{0}$ equals zero, thus obtaining the zero weak convergence of $\left(u_{n}\right)$. The associated H-measure is well known, and is combination of the Lebesgue measure (in $\mathbf{x}$ ) and the Dirac mass (in $\boldsymbol{\xi}$ ):

$$
\mu(\mathbf{x}, \boldsymbol{\xi})=\sum_{\mathbf{k}}\left|\hat{u}_{\mathbf{k}}\right|^{2} \delta_{\frac{\mathbf{k}}{|\mathbf{k}|}}(\boldsymbol{\xi}) \lambda(\mathbf{x}) .
$$

By using the above described procedure, we would like to express the limit of (2) in the case $p=4$. First, let us note that for a periodic function $u_{n}$, the action of the multiplier $\mathcal{A}_{\psi}$ results in a periodic function again:

$$
\mathcal{A}_{\psi} u_{n}(\mathbf{x})=\sum_{\mathbf{k}} \hat{u}_{\mathbf{k}} \psi(\mathbf{k}) e^{2 \pi i n \mathbf{k} \cdot \mathbf{x}},
$$

where we have used that the symbol of the above multiplier is a homogeneous function of order zero. Specially, function $v_{n}$ defined by (5) in this case takes the form

$$
v_{n}(\mathbf{x})=\left(\mathcal{A}_{\psi_{1}} u_{n}\right)\left(\mathcal{A}_{\psi_{2}} u_{n}\right)(\mathbf{x})=\sum_{\mathbf{j}, \mathbf{k}} \hat{u}_{\mathbf{j}} \hat{u}_{\mathbf{k}} \psi_{1}(\mathbf{j}) \psi_{2}(\mathbf{k}) e^{2 \pi i n(\mathbf{j}+\mathbf{k}) \cdot \mathbf{x}},
$$

and converges weakly to

$$
v(\mathbf{x})=\sum_{\mathbf{k}} \hat{u}_{\mathbf{k}} \hat{u}_{-\mathbf{k}} \psi_{1}(\mathbf{k}) \psi_{2}(-\mathbf{k}) .
$$

Similarly,

$$
\begin{aligned}
\overline{w_{n}}(\mathbf{x}) & =\left(\mathcal{A}_{\psi_{3}} u_{n}\right)\left(\mathcal{A}_{\psi_{4}} u_{n}\right)(\mathbf{x})=\sum_{\mathbf{l}, \mathbf{m}} \hat{u}_{\mathbf{l}} \hat{u}_{\mathbf{m}} \psi_{3}(\mathbf{l}) \psi_{4}(\mathbf{m}) e^{2 \pi i n(\mathbf{l}+\mathbf{m}) \cdot \mathbf{x}} \\
& \longrightarrow \bar{w}(\mathbf{x})=\sum_{\mathbf{l}} \hat{u}_{\mathbf{l}} \hat{u}_{-\mathbf{l}} \psi_{3}(\mathbf{l}) \psi_{4}(-\mathbf{l}) .
\end{aligned}
$$

The measure $\mu_{v w}$ determined by the sequences $\left(v_{n}-v\right)$ and $\left(w_{n}-w\right)$ then reads

$$
\mu_{v w}=\sum_{\substack{\mathbf{j}, \mathbf{k} \\ \mathbf{j}+\mathbf{k} \neq\{0\}}}\left(\sum_{\substack{\mathbf{l}, \mathbf{m} \\ \mathbf{l}+\mathbf{m}=-(\mathbf{j}+\mathbf{k})}} \hat{u}_{\mathbf{j}} \hat{u}_{\mathbf{k}} \hat{u}_{\mathbf{l}} \hat{u}_{\mathbf{m}} \psi_{1}(\mathbf{j}) \psi_{2}(\mathbf{k}) \psi_{3}(\mathbf{l}) \psi_{4}(\mathbf{m})\right) \delta_{\frac{\mathbf{j}+\mathbf{k}}{|\mathbf{j}+\mathbf{k}|}}(\boldsymbol{\xi}) \lambda(\mathbf{x}) .
$$

Taking into account the form of the limits $v$ and $w$, relation (3) implies

$$
\begin{aligned}
\lim _{n} & \int_{\mathbf{R}^{d}} \mathcal{A}_{\psi_{1}}\left(\varphi_{1} u_{n}\right)(\mathbf{x}) \cdot \ldots \cdot \mathcal{A}_{\psi_{4}}\left(\varphi_{4} u_{n}\right)(\mathbf{x}) d \mathbf{x} \\
& =\sum_{\substack{\mathbf{j}, \mathbf{k}, \mathbf{l}, \mathbf{m} \\
\mathbf{l}+\mathbf{m}=-(\mathbf{j}+\mathbf{k})}} \hat{u}_{\mathbf{j}} \hat{u}_{\mathbf{k}} \hat{u}_{\mathbf{l}} \hat{u}_{\mathbf{m}} \psi_{1}(\mathbf{j}) \psi_{2}(\mathbf{k}) \psi_{3}(\mathbf{l}) \psi_{4}(\mathbf{m}) \int_{\mathbf{R}^{d}} \varphi(\mathbf{x}) d \mathbf{x},
\end{aligned}
$$

where, let it be repeated, $\varphi=\prod_{i} \varphi_{i}$.

Similar approach can be applied for other values of $p$. Here we briefly comment the case $p=3$, as a prototype for odd values of $p$.

Note that due to additional regularity of sequence $\left(u_{n}\right)$ (bounded in $\mathrm{L}_{\mathrm{loc}}^{\infty}\left(\mathbf{R}^{d}\right)$, and thus in $\mathrm{L}_{\text {loc }}^{q}\left(\mathbf{R}^{d}\right)$, for any $q$ ), we can avoid splitting of the middle factor in (2) into two terms involving the square root, and define

$$
v_{n}=\mathcal{A}_{\psi_{1}} u_{n}, \quad \overline{w_{n}}=\left(\mathcal{A}_{\psi_{2}} u_{n}\right)\left(\mathcal{A}_{\psi_{3}} u_{n}\right)
$$


which both belong to $\mathrm{L}_{\text {loc }}^{2}\left(\mathbf{R}^{d}\right)$. Thus the associated measure is well defined and computing as above one gets

$$
\mu_{v w}=\sum_{\mathbf{k} \neq\{0\}}\left(\sum_{\substack{\mathbf{l}, \mathbf{m} \\ \mathbf{k}+\mathbf{l}+\mathbf{m}=0}} \hat{u}_{\mathbf{k}} \hat{u}_{\mathbf{l}} \hat{u}_{\mathbf{m}} \psi_{1}(\mathbf{k}) \psi_{2}(\mathbf{l}) \psi_{3}(\mathbf{m})\right) \delta_{\frac{\mathbf{k}}{|\mathbf{k}|}}(\boldsymbol{\xi}) \lambda(\mathbf{x}) .
$$

and

$$
\begin{aligned}
\lim _{n} & \int_{\mathbf{R}^{d}} \mathcal{A}_{\psi_{1}}\left(\varphi_{1} u_{n}\right)(\mathbf{x}) \cdot \ldots \cdot \mathcal{A}_{\psi_{3}}\left(\varphi_{3} u_{n}\right)(\mathbf{x}) d \mathbf{x} \\
& =\sum_{\substack{\mathbf{k}, \mathbf{l}, \mathbf{m} \\
\mathbf{k}+\mathbf{l}+\mathbf{m}=0}} \hat{u}_{\mathbf{k}} \hat{u}_{\mathbf{l}} \hat{u}_{\mathbf{m}} \psi_{1}(\mathbf{k}) \psi_{2}(\mathbf{l}) \psi_{3}(\mathbf{m}) \int_{\mathbf{R}^{d}} \varphi(\mathbf{x}) d \mathbf{x}
\end{aligned}
$$

Similarly, the last formula is now easily extend to an arbitrary integer $p \geq 2$, providing the following result.

Theorem 5. Let $\left(u_{n}\right)$ be a bounded sequence of periodic functions in $\mathrm{L}_{\mathrm{loc}}^{\infty}\left(\mathbf{R}^{d}\right)$ defined by (8), with the mean value zero. Then for any $p \in \mathbf{N}$ and any choice of test functions $\varphi_{i} \in \mathrm{C}_{c}\left(\mathbf{R}^{d}\right), \psi_{i} \in \mathrm{C}^{d}\left(\mathrm{~S}^{d-1}\right), i=1 . . p$ it holds

$$
\lim _{n} \int_{\mathbf{R}^{d}} \mathcal{A}_{\psi_{1}}\left(\varphi_{1} u_{n}\right)(\mathbf{x}) \cdot \ldots \cdot \mathcal{A}_{\psi_{p}}\left(\varphi_{p} u_{n}\right)(\mathbf{x}) d \mathbf{x}=\sum_{\substack{\mathbf{k}_{i} \in \mathbf{Z}^{d}, \sum_{i} \mathbf{k}_{i}=0}}\left(\prod_{i=1}^{p} \hat{u}_{\mathbf{k}_{i}} \psi_{i}\left(\mathbf{k}_{i}\right)\right) \int_{\mathbf{R}^{d}} \varphi(\mathbf{x}) d \mathbf{x}
$$

where $\varphi=\prod_{i=1}^{p} \varphi_{i}$.

Remark 6 . The last theorem easily generalises to a case when each factor of the integrand in (13) is associated to a different sequence $\left(u_{n}^{i}\right)_{n}, i=1 . . p$, just by adjusting the Fourier coefficients on the right hand side.

Note that the last theorem also incorporates the expression (9) for an H-measure associated to a sequence of periodic functions.

\section{Computation of higher order CORRECtion terms in SMAll Amplitude HOMOGENISATION}

3.1. Small amplitude homogenisation. We consider a sequence of elliptic problems:

$$
\left\{\begin{aligned}
-\operatorname{div}\left(\mathbf{A}^{n} \nabla u^{n}\right) & =f \in \mathrm{H}^{-1}(\Omega) \\
u^{n} & \in \mathrm{H}_{0}^{1}(\Omega),
\end{aligned}\right.
$$

where $\Omega \subseteq \mathbf{R}^{d}$ is an open, bounded domain.

If the coefficients $\mathbf{A}^{n}$ are taken from the set (with $0<\alpha<\beta$ )

$\mathcal{M}(\alpha, \beta ; \Omega):=\left\{\mathbf{A} \in \mathrm{L}^{\infty}\left(\Omega ; \mathcal{L}\left(\mathbf{R}^{d} ; \mathbf{R}^{d}\right)\right): \mathbf{A}(\mathbf{x}) \boldsymbol{\xi} \cdot \boldsymbol{\xi} \geq \alpha|\boldsymbol{\xi}|^{2} \& \mathbf{A}^{-1}(\mathbf{x}) \boldsymbol{\xi} \cdot \boldsymbol{\xi} \geq \frac{1}{\beta}|\boldsymbol{\xi}|^{2}(\right.$ a.e. $\left.\mathbf{x} \in \Omega)\right\}$

we have that (after extracting a non-relabelled subsequence) $\mathbf{A}^{n} \stackrel{H}{\longrightarrow} \mathbf{A}^{\infty} \in \mathcal{M}(\alpha, \beta ; \Omega)$

(e.g. [18, Theorem 6.5]). The above H-convergence means that for any choice of 
$f \in \mathrm{H}^{-1}(\Omega)$, the sequence of solutions to (14) satisfies:

$$
\begin{gathered}
u^{n} \longrightarrow u^{\infty} \quad \text { in } \mathrm{H}_{0}^{1}(\Omega) \\
\mathbf{A}^{n} \nabla u^{n} \longrightarrow \mathbf{A}^{\infty} \nabla u^{\infty} \quad \text { in } \mathrm{L}^{2}(\Omega),
\end{gathered}
$$

where $u^{\infty}$ is the solution of (14) with $\infty$ instead of $n$.

For small amplitude homogenisation we consider $\mathbf{A}^{n}$ to be a perturbation of a constant:

$$
\mathbf{A}_{\gamma}^{n}(t, \mathbf{x})=\mathbf{A}_{0}+\gamma \mathbf{A}_{1}^{n}(t, \mathbf{x})+\gamma^{2} \mathbf{A}_{2}^{n}(t, \mathbf{x})+\gamma^{3} \mathbf{A}_{3}^{n}(t, \mathbf{x})+o\left(\gamma^{3}\right),
$$

where $\mathbf{A}_{i}^{n} \stackrel{*}{\longrightarrow} \mathbf{0}$ in $\mathrm{L}^{\infty}(\Omega)$ for any $i \geq 1$.

Assuming that $\mathbf{A}_{0} \in \mathcal{M}(\alpha, \beta ; \Omega)$, we have that (for small values of $\gamma$ )

$$
\mathbf{A}_{\gamma}^{n} \stackrel{H}{\longrightarrow} \mathbf{A}_{\gamma}^{\infty}=\mathbf{A}_{0}+\gamma \mathbf{A}_{1}^{\infty}(t, \mathbf{x})+\gamma^{2} \mathbf{A}_{2}^{\infty}(t, \mathbf{x})+\gamma^{3} \mathbf{A}_{3}^{\infty}(t, \mathbf{x})+o\left(\gamma^{3}\right),
$$

where the homogenised limit $\mathbf{A}_{\gamma}^{\infty}$ is measurable in $\mathbf{x}$ and analytic in $\gamma$ (for details on small amplitude homogenisation consult [18, Chapter 29]).

The components of the limit $\mathbf{A}_{\gamma}^{\infty}$ up to the second order of $\gamma$ are given explicitly by means of H-measures. More precisely, the following theorem holds (cf. [17, Theorem 4.2]).

Theorem 7. The effective diffusion tensor $\mathbf{A}_{\gamma}^{\infty}$ satisfies

$$
\mathbf{A}_{\gamma}^{\infty}=\mathbf{A}_{0}+\gamma^{2} \mathbf{A}_{2}^{\infty}(t, \mathbf{x})+o\left(\gamma^{2}\right)
$$

where the second order correction is given by

$$
\int_{\Omega}\left(\mathbf{A}_{2}^{\infty}\right)_{i j}(\mathbf{x}) \phi(\mathbf{x}) d \mathbf{x}=-\sum_{k, l}\left\langle\mu_{11}^{i k l j}, \phi \frac{\xi_{k} \xi_{l}}{\mathbf{A}_{0} \boldsymbol{\xi} \cdot \boldsymbol{\xi}}\right\rangle
$$

with $\boldsymbol{\mu}_{11}$ standing for an $H$-measure (with four indices) associated to $\mathbf{A}_{1}^{n}$.

3.2. Expressing higher order correction terms. The goal of the following study is to use the approach presented in Section 2 in order to construct explicit expressions for higher order homogenised terms. We shall see that such obtained formulæ will include H-measures associated to (the powers of) the lower order perturbations. Here we follow the approach presented firstly in [17] for elliptic problems (for the parabolic version see [4]).

Fix a function $u \in \mathrm{H}_{0}^{1}(\Omega)$, and denote by $u_{\gamma}^{n}$ the solution of

$$
-\operatorname{div}\left(\mathbf{A}_{\gamma}^{n} \nabla u_{\gamma}^{n}\right)=-\operatorname{div}\left(\mathbf{A}_{\gamma}^{\infty} \nabla u\right)
$$

Because of H-convergence, we have that $u_{\gamma}^{n} \longrightarrow u$ (in $\mathrm{H}_{0}^{1}(\Omega)$ ) and that $\mathrm{D}_{\gamma}^{n}:=$ $\mathbf{A}_{\gamma}^{n} \nabla u_{\gamma}^{n} \longrightarrow \mathbf{A}_{\gamma}^{\infty} \nabla u\left(\right.$ in $\mathrm{L}^{2}(\Omega)$ ). After writing the expansions in powers of $\gamma$ :

$$
u_{\gamma}^{n}=u_{0}^{n}+\gamma u_{1}^{n}+\gamma^{2} u_{2}^{n}+o\left(\gamma^{2}\right), \quad \mathrm{D}_{\gamma}^{n}=\mathrm{D}_{0}^{n}+\gamma \mathrm{D}_{1}^{n}+\gamma^{2} \mathrm{D}_{2}^{n}+o\left(\gamma^{2}\right),
$$

we see that $u_{0}^{n} \longrightarrow u$ and $u_{i}^{n} \longrightarrow 0$ for $i \geq 1$, while it remains to calculate the limits of $\mathrm{D}_{i}^{n}$.

Equating the terms with equal powers of $\gamma$ we get $\nabla u_{0}^{n}=\nabla u, \mathrm{D}_{0}^{n}=\mathbf{A}_{0} \nabla u$ (with $\gamma^{0}$ ); and $\mathbf{D}_{1}^{n}=\mathbf{A}_{0} \nabla u_{1}^{n}+\mathbf{A}_{1}^{n} \nabla u \longrightarrow 0$ (with $\gamma^{1}$ ). On the other hand, $\mathrm{D}_{1}^{n}$ converges to $\mathbf{A}_{1}^{\infty} \nabla u$ (the term in expansion of $\mathbf{A}_{\gamma}^{\infty} \nabla u$ with power of $\gamma$ equal 1 ), which, by varying $u$, gives that $\mathbf{A}_{1}^{\infty}=\mathbf{0}$.

For the quadratic term we have:

$$
\mathrm{D}_{2}^{n}=\mathbf{A}_{0} \nabla u_{2}^{n}+\mathbf{A}_{1}^{n} \nabla u_{1}^{n}+\mathbf{A}_{2}^{n} \nabla u \longrightarrow \lim _{n} \mathbf{A}_{1}^{n} \nabla u_{1}^{n}=\mathbf{A}_{2}^{\infty} \nabla u,
$$


By expansion of (17) and taking terms with $\gamma^{1}$, observe that $u_{1}^{n}$ satisfies the problem (14) with $\mathbf{A}^{n}=\mathbf{A}_{0}$ and the right hand side equal to $\operatorname{div}\left(\mathbf{A}_{1}^{n} \nabla u\right)$. As $\mathbf{A}_{0}$ is a constant matrix, application of the Fourier transform yields

$$
\widehat{\nabla u_{1}^{n}}(\boldsymbol{\xi})=-\frac{(\boldsymbol{\xi} \otimes \boldsymbol{\xi})\left(\widehat{\mathbf{A}_{1}^{n} \nabla u}\right)(\boldsymbol{\xi})}{\mathbf{A}_{0} \boldsymbol{\xi} \cdot \boldsymbol{\xi}}
$$

or equivalently

$$
\nabla u_{1}^{n}(\mathbf{x})=-\mathcal{A}_{\Psi}\left(\mathbf{A}_{1}^{n} \nabla u\right)(\mathbf{x}),
$$

where $\mathcal{A}_{\psi}$ is the multiplier with the symbol $\Psi(\boldsymbol{\xi})=\frac{\boldsymbol{\xi} \otimes \boldsymbol{\xi}}{\mathbf{A}_{0} \boldsymbol{\xi} \cdot \boldsymbol{\xi}}$.

Taking into account (18), one gets that the second order correction $\mathbf{A}_{2}$ is expressed via limit of the quadratic term in $\mathbf{A}_{1}^{n}$, i.e. via a corresponding $\mathrm{H}$-measure, which is essentially the result of Theorem 7 .

In order to find the cubic term, note that

$\mathrm{D}_{3}^{n}=\mathbf{A}_{0} \nabla u_{3}^{n}+\mathbf{A}_{1}^{n} \nabla u_{2}^{n}+\mathbf{A}_{2}^{n} \nabla u_{1}^{n}+\mathbf{A}_{3}^{n} \nabla u \longrightarrow \lim _{n}\left(\mathbf{A}_{1}^{n} \nabla u_{2}^{n}+\mathbf{A}_{2}^{n} \nabla u_{1}^{n}\right)=\mathbf{A}_{3}^{\infty} \nabla u$,

where we have taken into account zero weak convergence of $\left(\mathbf{A}_{i}^{n}\right)$ and $\left(u_{i}^{n}\right)$ for $i \geq 1$.

On the other hand, by equating the terms with $\gamma^{2}$ in the expansion of the relation (17), one gets for $u_{2}^{n}$

$$
-\operatorname{div}\left(\mathbf{A}_{0} \nabla u_{2}^{n}\right)=\operatorname{div}\left(\mathbf{A}_{2}^{n} \nabla u+\mathbf{A}_{1}^{n} \nabla u_{1}^{n}-\mathbf{A}_{2}^{\infty} \nabla u\right) \longrightarrow 0,
$$

where the convergence of the right hand side follows from (18).

By applying the Fourier transform, similarly as in (19) one gets

$$
\nabla u_{2}^{n}(\mathbf{x})=-\mathcal{A}_{\Psi}\left(\mathbf{A}_{2}^{n} \nabla u+\mathbf{A}_{1}^{n} \nabla u_{1}^{n}-\mathbf{A}_{2}^{\infty} \nabla u\right)(\mathbf{x}) .
$$

Putting the last expression together with (19) in (20), integration and multiplication by a test function $\varphi \in \mathrm{C}_{c}^{\infty}(\Omega)$ yields

$$
\int_{\Omega} \varphi \mathbf{A}_{3}^{\infty} \nabla u d \mathbf{x}=\lim _{n} \int_{\Omega} \varphi\left(-\mathbf{A}_{1}^{n} \mathcal{A}_{\Psi} \mathbf{A}_{2}^{n} \nabla u-\mathbf{A}_{2}^{n} \mathcal{A}_{\Psi} \mathbf{A}_{1}^{n} \nabla u+\mathbf{A}_{1}^{n} \mathcal{A}_{\Psi}\left(\mathbf{A}_{1}^{n} \mathcal{A}_{\Psi} \mathbf{A}_{1}^{n} \nabla u\right)\right) d \mathbf{x}
$$

The limits of the first two terms on the right hand side are expressed via $\mathrm{H}$-measures determined by functions $\mathbf{A}_{1}^{n}$ and $\mathbf{A}_{2}^{n}$. The last term in (21) is a cubic term in $\mathbf{A}_{1}^{n}$. As applications of $\mathrm{H}$-measures so far have been constrained to quadratic expressions, for that reason no expression for the third order correction has been obtained. In the sequel we shall overcome the restriction and calculate its limit by means of Theorem 2.

We analyse the quadratic terms first. Carefully expanding the product by components we obtain

$$
\lim _{n} \int_{\Omega} \varphi\left(\mathbf{A}_{1}^{n} \mathcal{A}_{\Psi} \mathbf{A}_{2}^{n}+\mathbf{A}_{2}^{n} \mathcal{A}_{\Psi} \mathbf{A}_{1}^{n}\right) \nabla u d \mathbf{x}=\left\langle 2 \Re \boldsymbol{\mu}_{12}, \varphi \frac{\boldsymbol{\xi} \otimes \boldsymbol{\xi} \otimes \nabla u}{\mathbf{A}_{0} \boldsymbol{\xi} \cdot \boldsymbol{\xi}}\right\rangle,
$$

where $\boldsymbol{\mu}_{12}$ is an off-diagonal block term of the H-measure associated to $\left(\mathbf{A}_{1}^{n}, \mathbf{A}_{2}^{n}\right)$ a measure with four indices (the first of them not being contracted above).

As for the cubic term, observe that by using an pseudodifferential calculus identity $\int\left(\mathcal{A}_{\psi} u\right) v d x=\int u \mathcal{A}_{\tilde{\psi}} v d x$ (where $\tilde{\psi}$ denotes the change of the argument sign, i.e. $\tilde{\psi}(\xi)=\psi(-\xi))$ it can be rewritten as

$$
\lim _{n} \int_{\Omega}\left(\mathcal{A}_{\Psi}\left(\varphi \mathbf{A}_{1}^{n}\right)^{\top}\right)^{\top} \mathbf{A}_{1}^{n} \mathcal{A}_{\Psi} \mathbf{A}_{1}^{n} \nabla u d \mathbf{x}
$$


where symmetry and evenness of $\Psi$ has been taken into account. Denoting

$$
\mathbf{V}^{n}=\left(\mathcal{A}_{\Psi}\left(\varphi \mathbf{A}_{1}^{n}\right)^{\top}\right)^{\top}, \quad \mathbf{W}^{n}=\mathbf{A}_{1}^{n} \mathcal{A}_{\Psi} \mathbf{A}_{1}^{n},
$$

Theorem 2 gives that the $i$-th component of (22) equals $\sum_{j, k}\left\langle\mu_{v^{i k} w^{k j}}, \varphi \partial_{j} u \otimes 1\right\rangle$ $\left(\mu_{v^{i k} w^{k j}}\right.$ denotes appropriate component of the four-index H-measure $\boldsymbol{\mu}_{V W}$ associated to $\left.\left(\mathbf{V}^{n}, \overline{\mathbf{W}}^{n}\right)\right)$. Note that we omit the last term appearing in (3), as the (zero) weak convergence assumption on coefficients $\mathbf{A}_{1}^{n}$ implies the weak limit of $\mathbf{V}^{n}$ equals zero. By varying function $u \in \mathrm{C}^{1}(\Omega)$ (e.g. choosing $\nabla u$ constant on $\omega$, where $\omega \Subset \Omega)$ we finally obtain the following result.

Theorem 8. The third order correction of the effective diffusion tensor $\mathbf{A}_{\gamma}^{\infty}$ defined in (16) is given by

$$
\int_{\Omega}\left(\mathbf{A}_{3}^{\infty}\right)^{i j} \varphi d \mathbf{x}=-\left\langle 2 \Re \boldsymbol{\mu}_{12}^{i j}, \varphi \frac{\boldsymbol{\xi} \otimes \boldsymbol{\xi}}{\mathbf{A}_{0} \boldsymbol{\xi} \cdot \boldsymbol{\xi}}\right\rangle+\left\langle\operatorname{tr} \boldsymbol{\mu}_{V W}^{i j}, \varphi \otimes 1\right\rangle,
$$

where $\boldsymbol{\mu}_{12}^{i j}$ denotes the matrix H-measure determined by sequences $\left(\mathbf{A}_{1}^{n}\right)$ and $\left(\mathbf{A}_{2}^{n}\right)$ with components $\left(\boldsymbol{\mu}_{12}^{i j}\right)^{k l}=\mu_{12}^{i k l j}$, and similarly for the H-measure $\boldsymbol{\mu}_{V W}^{i j}$, whose generating sequences are given by (23).

Remark 9. Although not given explicitly, the last term in (24) depends on $\Psi=$ $\frac{\boldsymbol{\xi} \otimes \boldsymbol{\xi}}{\mathbf{A}_{0} \boldsymbol{\xi} \cdot \boldsymbol{\xi}}$ indeed, as both sequences $\left(V^{n}\right),\left(W^{n}\right)$, as well as the associated H-measure depend on it. This dependence is more apparent if a periodic setting is considered, which is the subject of the next subsection.

Similar procedure as in the above can be applied in order to express further order correction terms of the effective diffusion tensor. Derivation for the fourth order term is sketched in the next subsection under periodicity assumption.

3.3. Periodic setting. This subsection is devoted to a special, but important case of periodic coefficients in (14). By employing techniques of Section 2 we derive explicit formulæ for higher order terms expressed by Fourier coefficients of $\mathbf{A}^{n}$.

We suppose the coefficients in (15) to be of the form

$$
\mathbf{A}_{i}^{n}(n \mathbf{x})=\mathbf{A}_{i}(n \mathbf{x})=\sum_{\mathbf{k} \in \mathbf{Z}^{d}} \hat{\mathbf{A}}_{i, \mathbf{k}} e^{2 \pi i n \mathbf{k} \cdot \mathbf{x}}, \quad i \in \mathbf{N}
$$

where (just to simplify calculations) each $\mathbf{A}_{i}$ is symmetric with the zero mean value.

The measure associated to $\left(\mathbf{A}_{1}^{n}, \mathbf{A}_{2}^{n}\right)$ takes the form

$$
\boldsymbol{\mu}_{12}=\sum_{\mathbf{k}} \hat{\mathbf{A}}_{1, \mathbf{k}} \otimes \hat{\mathbf{A}}_{2,-\mathbf{k}} \delta \frac{\mathbf{k}}{|\mathbf{k}|}(\boldsymbol{\xi}) \lambda(\mathbf{x}) .
$$

Thus the first term on the right hand side of (24) equals

$$
-2 \sum_{\mathbf{k}} \frac{1}{\mathbf{A}_{0} \mathbf{k} \cdot \mathbf{k}} \int_{\Omega}\left(\hat{\mathbf{A}}_{1, \mathbf{k}} \mathbf{k}\right) \otimes\left(\hat{\mathbf{A}}_{2,-\mathbf{k}} \mathbf{k}\right) \varphi(\mathbf{x}) d \mathbf{x} .
$$

As for the second term one uses the formula (11) for an H-measure associated to cubic terms (in $\mathbf{A}_{1}^{n}$ here). Thus the trace of $\boldsymbol{\mu}_{V W}$ (the trace being taken with 
respect to the middle two components) equals

$$
\operatorname{tr} \boldsymbol{\mu}_{V W}=\sum_{\substack{\mathbf{k}, \mathbf{l}, \mathbf{m} \\ \mathbf{k}+\mathbf{l}+\mathbf{m}=0}} \frac{\hat{\mathbf{A}}_{1, \mathbf{l}} \mathbf{k} \cdot \mathbf{m}}{\left(\mathbf{A}_{0} \mathbf{k} \cdot \mathbf{k}\right)\left(\mathbf{A}_{0} \mathbf{m} \cdot \mathbf{m}\right)}\left(\hat{\mathbf{A}}_{1, \mathbf{k}} \mathbf{k}\right) \otimes\left(\hat{\mathbf{A}}_{1, \mathbf{m}} \mathbf{m}\right) \delta_{\frac{\mathbf{k}}{|\mathbf{k}|}}(\boldsymbol{\xi}) \lambda(\mathbf{x}),
$$

and for the third order correction we obtain the explicit expression

$$
\mathbf{A}_{3}^{\infty}=\sum_{\mathbf{k} \in \mathbf{Z}^{d}} \frac{1}{\mathbf{A}_{0} \mathbf{k} \cdot \mathbf{k}}\left(\hat{\mathbf{A}}_{1, \mathbf{k}} \mathbf{k}\right) \otimes\left(-2 \hat{\mathbf{A}}_{2,-\mathbf{k}} \mathbf{k}+\sum_{\substack{\mathbf{1}, \mathbf{m} \in \mathbf{Z}^{d} \\ \mathbf{k}+\mathbf{l}+\mathbf{m}=0}} \frac{\hat{\mathbf{A}}_{1, \mathbf{l}} \mathbf{k} \cdot \mathbf{m}}{\mathbf{A}_{0} \mathbf{m} \cdot \mathbf{m}} \hat{\mathbf{A}}_{1, \mathbf{m}} \mathbf{m}\right) .
$$

Just for comparison, here we provide an analogous expression for $\mathbf{A}_{2}^{\infty}$ obtained by means of Theorem 7:

$$
\mathbf{A}_{2}^{\infty}=-\sum_{\mathbf{k} \in \mathbf{Z}^{d}} \frac{1}{\mathbf{A}_{0} \mathbf{k} \cdot \mathbf{k}}\left(\hat{\mathbf{A}}_{1, \mathbf{k}} \mathbf{k}\right) \otimes \hat{\mathbf{A}}_{1,-\mathbf{k}} \mathbf{k}
$$

By virtue of Theorem 5 derivation of expressions for higher order terms goes similarly, just involving more lengthy computations of linear algebra. Here we briefly provide the result for $\mathbf{A}_{4}^{\infty}$.

Similarly as (21) one obtains

$$
\begin{aligned}
\int_{\Omega} \mathbf{A}_{4}^{\infty} \nabla u d \mathbf{x} & =\lim _{n} \int_{\Omega}\left(-\mathbf{A}_{3}^{n} \mathcal{A}_{\Psi} \mathbf{A}_{1}^{n} \nabla u-\mathbf{A}_{2}^{n} \mathcal{A}_{\Psi} \mathbf{A}_{2}^{n} \nabla u-\mathbf{A}_{1}^{n} \mathcal{A}_{\Psi} \mathbf{A}_{3}^{n} \nabla u\right. \\
& +\left(\mathcal{A}_{\Psi} \mathbf{A}_{1}^{n}\right)^{\top} \mathbf{A}_{1}^{n} \mathcal{A}_{\Psi} \mathbf{A}_{2}^{n} \nabla u+\left(\mathcal{A}_{\Psi} \mathbf{A}_{1}^{n}\right)^{\top} \mathbf{A}_{2}^{n} \mathcal{A}_{\Psi} \mathbf{A}_{1}^{n} \nabla u \\
& \left.+\left(\mathcal{A}_{\Psi} \mathbf{A}_{2}^{n}\right)^{\top} \mathbf{A}_{1}^{n} \mathcal{A}_{\Psi} \mathbf{A}_{1}^{n} \nabla u-\left(\mathcal{A}_{\Psi} \mathbf{W}^{n}\right)^{\top} \mathbf{W}^{n} \nabla u\right) d \mathbf{x}
\end{aligned}
$$

where $\mathbf{W}^{n}$ is given by (23).

The first three terms on the right hand side are quadratic terms whose limit is expressed via standard H-measures. The next three are cubic ones, and the limit is obtained by virtue of relation (12), similarly as it was done above for $\mathbf{A}_{3}^{\infty}$. The last one is a quartic term in $\mathbf{A}_{1}^{n}$ (as $\mathbf{W}^{n}$ are quadratic expressions of $\mathbf{A}_{1}^{n}$ ), which is treated by means of formula (10). Performing some tedious, but mostly elementary computations one gets

$$
\begin{aligned}
& \mathbf{A}_{4}^{\infty}= \sum_{\mathbf{k} \in \mathbf{Z}^{d}} \frac{1}{\mathbf{A}_{0} \mathbf{k} \cdot \mathbf{k}}\left(-2\left(\hat{\mathbf{A}}_{1, \mathbf{k}} \mathbf{k}\right) \otimes \hat{\mathbf{A}}_{3,-\mathbf{k}} \mathbf{k}-\left(\hat{\mathbf{A}}_{2, \mathbf{k}} \mathbf{k}\right) \otimes\left(\hat{\mathbf{A}}_{2},-\mathbf{k}\right)\right. \\
&+ \sum_{\substack{\mathbf{1}, \mathbf{m} \in \mathbf{Z}^{d} \\
\mathbf{k}+\mathbf{l}+\mathbf{m}=0}} \frac{1}{\mathbf{A}_{0} \mathbf{m} \cdot \mathbf{m}}\left(\hat{\mathbf{A}}_{1, \mathbf{l}} \mathbf{k} \cdot \mathbf{m}\left(\hat{\mathbf{A}}_{1, \mathbf{k}} \mathbf{k}\right) \otimes\left(\hat{\mathbf{A}}_{2, \mathbf{m}} \mathbf{m}\right)+\hat{\mathbf{A}}_{2, \mathbf{l}} \mathbf{k} \cdot \mathbf{m}\left(\hat{\mathbf{A}}_{1, \mathbf{k}} \mathbf{k}\right) \otimes\left(\hat{\mathbf{A}}_{1, \mathbf{m}} \mathbf{m}\right)\right. \\
& \quad+\hat{\mathbf{A}}_{1, \mathbf{l}} \mathbf{k} \cdot \mathbf{m}\left(\hat{\mathbf{A}}_{2, \mathbf{k}} \mathbf{k}\right) \otimes\left(\hat{\mathbf{A}}_{1, \mathbf{m}} \mathbf{m}\right) \\
&\left.\left.-\sum_{\substack{\mathbf{j} \in \mathbf{Z}^{d} \\
\mathbf{l}+\mathbf{m}=-(\mathbf{j}+\mathbf{k})}} \frac{1}{\mathbf{A}_{0}(\mathbf{j}+\mathbf{k}) \cdot(\mathbf{j}+\mathbf{k})}\left(\hat{\mathbf{A}}_{1, \mathbf{j}} \mathbf{k}+\hat{\mathbf{A}}_{1, \mathbf{l}} \mathbf{m}\right) \cdot(\mathbf{j}+\mathbf{k})\left(\hat{\mathbf{A}}_{1, \mathbf{k}} \mathbf{k}\right) \otimes\left(\hat{\mathbf{A}}_{1, \mathbf{m}} \mathbf{m}\right)\right)\right)
\end{aligned}
$$


Remark 10. - The above results are easily generalised to the case when $\mathbf{A}_{i}^{n} \stackrel{*}{\longrightarrow} \mathbf{A}_{i} \neq \mathbf{0}$, with correction terms entering expression for the homogenised limit $\mathbf{A}_{\gamma}^{\infty}$.

- In analysis of small amplitude homogenisation we considered diffusion coefficients $\mathbf{A}_{n}$ to be perturbations of a constant. This was important in order to explicitly express $\nabla u_{i}^{n}$ via a given function $u$ (relation (19) etc). However, one can try to generalise result to a variable $\mathbf{A}_{0}$ by justifying and following suggestions given in [8] or [17], partially based on the localisation principle for H-measures. Here it is important that the measures analysed in previous section obey the same principle in accordance to subsection 2.2, making potential generalisation feasible.

\section{Conclusion}

In the paper we have developed a method for expressing limits of non-quadratic terms by means of original H-measures, followed by application to the small amplitude homogenisation problem for a stationary diffusion equation. The method provides higher order correction terms of the effective diffusion tensor expressed by $\mathrm{H}$-measures associated to non-quadratic terms in a general, non-periodic setting. Explicit formulæ in terms of Fourier coefficients are obtained under periodicity assumption.

A similar approach can be also conducted in the case of non-stationary diffusion problems by means of parabolic H-measures $[4,6]$. The latter represent a generalisation of the tool that takes into account the difference between the time and space variables, intrinsic to parabolic type problems.

The corresponding analysis, as well as the explicit formula for the second order homogenised term has been provided firstly by [4] for the periodic functions, while non-periodic generalisations are given in [8]. Results and the approach presented in Section 2 of expressing non-quadratic limits via microlocal defect measures extend easily to parabolic $\mathrm{H}$-measures as well. Thus the third order homogenised terms for a non-stationary diffusion problem can be easily obtained by slightly adapting the proof of Theorem 8, and similarly for higher orders.

The presented method for expressing non-quadratic terms can also be generalised to recently introduced multiscale H-measures $[19,3]$. Unlike the original ones, multiscale variant are capable of distinguishing sequences with different frequencies. Thus, their application in expressing higher order terms in small amplitude homogenisation makes full sense in the case of coefficients $\mathbf{A}_{i}^{n}$ oscillating on various scales.

The method developed in this paper paves the path for more precise analysis of other problems involving small amplitude homogenisation $[1,2,12]$ in which the analysis so far has been conducted by means of H-measures up to the second order expansion. It is important to notice that in all these papers sequences describing the limit terms posses higher regularity than the one required by the original definition of $\mathrm{H}$-measures ( $\mathrm{L}^{\infty}$ instead of merely $\mathrm{L}^{2}$ one) and fit within the setting of the presented approach, thus making its potential applications feasible.

\section{ACKNOWLEDGEMENTS}

This work was supported in part by the Croatian Science Foundation under Grant 9780 WeConMApp. 


\section{Disclosure STATEMENT}

The author declares that he has no conflict of interest.

\section{REFERENCES}

[1] G. Allaire, S. Gutiérrez, Optimal Design in Small Amplitude Homogenization, Math. Model. Numer. Anal. 41 (2007) 543-574.

[2] G. Allaire, A. Kelly, Optimal Design of Low-Contrast Two Phase Structures for the Wave Equation, Math. Models Methods Appl. Sci. 21(7) (2011) 1499-1538.

[3] N. Antonić, M. Erceg, M. Lazar, Localisation principle for one-scale H-measures, submitted.

[4] N. Antonić, M. Lazar, H-measures and variants applied to parabolic equations, J. Math. Anal. Appl. 343 (2008) 207-225.

[5] N. Antonić, M. Lazar, Parabolic variant of H-measures in homogenisation of a model problem based on Navier-Stokes equation, Nonlinear Anal. B: Real World Appl. 11 (2010) 45004512.

[6] N. Antonić, M. Lazar, Parabolic H-measures, J. Funct. Anal. 265 (2013) 1190-1239.

[7] N. Antonić, D. Mitrovic, H-distributions - an extension of the H-measures in $L^{p}-L^{q}$ setting, Abstr. Appl. Anal. 2011 (2011), 12 pp.

[8] N. Antonić, M. VRdoljak, Parabolic H-convergence and small-amplitude homogenisation, Appl. Analysis 88 (2009) 1493-1508.

[9] B. Dehman, M. Léautaud and J. Le Rousseau, Controllability of two coupled wave equations on a compact manifold. Arch. Rational Mech. Anal. 211(1) (2014) 113-187.

[10] P. GÉrard, Microlocal defect measures, Comm. Partial Diff. Eq. 16 (1991) 1761-1794.

[11] L. Grafakos, Classical Fourier Analysis, Graduate Text in Mathematics 249, 2008 Springer Science and Business Media, LLC

[12] S. GutiÉrrez, J. Mura Small amplitude homogenization applied to inverse problems Comput Mech. 41 (2008) 699-706.

[13] M. Lazar, D. Mitrović, Velocity averaging - a general framework, Dyn. Partial Differ. Equ. 9 (2012) 239-260.

[14] M. Lazar, D. Mitrović, On an extension of a bilinear functional on $L^{p}\left(R^{d}\right) \times E$ to a Bochner space with an application to velocity averaging, C. R. Acad. Sci. Paris Ser. I 351 (2013), 261-26.

[15] M. Lazar, D. Mitrović, Optimal velocity averaging in a degenerate elliptic setting, submitted

[16] M. LAZAR, E. ZuAZuA, Averaged control and observation of parameter-depending wave equations, C. R. Acad. Sci. Paris, Ser. I 352 (2014) 497-502.

[17] L. TARTAR, H-measures, a new approach for studying homogenisation, oscillation and concentration effects in PDEs, Proc. Roy. Soc. Edinburgh. Sect. A 115(3-4) (1990) 193-230.

[18] L. TARTAR, The general theory of homogenization: A personalized introduction, Springer, 2009.

[19] L. Tartar, Multi-scale H-measures, Discrete Cont. Dyn. S. Ser. S 8 (2015) 77-90.

Martin Lazar, University of Dubrovnik, Department of Electrical Engineering and Computing, Ćira Carića 4, 20000 Dubrovnik, Croatia

E-mail address: martin.lazar@unidu.hr 